\title{
The effects of electric fields on wind driven particulate detachment
}

\author{
C. Holstein-Rathlou, J.P. Merrison, C.F. Brædstrup, and P. Nornberg
}

Mars Simulation Laboratory, Department of Physics and Astronomy, Aarhus University, DK-8000 Aarhus C, Denmark

The generation of electric fields close to the surface during sand and dust transport events occurs as a consequence of contact electrification of the sand and dust particulates. Albeit a well documented phenomena the effects of electric fields on the wind driven detachment and transport of granular materials is not well understood.

To improve our understanding of the interaction of naturally occurring electric fields upon the transport of sand (and dust) within Aeolian events we have investigated the influence of the electrical conductivity of the surface on the wind driven detachment threshold for sand sized particulates at a surface. Small circular deposits of granular material were placed upon a surface in a parallel plate electrode system and the entire setup located within a re-circulating wind tunnel. This allowed for the application of various electric fields and wind speeds.

The study has demonstrated that there is a qualitative difference in the behaviour of the wind driven detachment threshold for an insulating surface as compared to a conductive surface. 